$\curvearrowright$

PERSONNEL ASSESSMENT

A N D DE CISIONS
Personnel Assessment and

Decisions

Volume 6

Issue 2 Applications of Judgment and Decision

Making to Problems in Personnel Assessment

Article 4

2020

\title{
Does Feedback Increase Decision Aid Use Among Hiring Professionals?
}

Aneeqa Thiele

Middle Tennessee State University

Alexander T. Jackson

Middle Tennessee State University

Stacey M. Stremic

Middle Tennessee State University

Satoris S. Howes

Oregon State University - Cascades Campus

Follow this and additional works at: https://scholarworks.bgsu.edu/pad

Part of the Human Resources Management Commons, Industrial and Organizational Psychology

Commons, and the Other Psychology Commons

How does access to this work benefit you? Let us know!

\section{Recommended Citation}

Thiele, Aneeqa; Jackson, Alexander T.; Stremic, Stacey M.; and Howes, Satoris S. (2020) "Does Feedback Increase Decision Aid Use Among Hiring Professionals?," Personnel Assessment and Decisions: Number 6 : Iss. 2 , Article 4.

DOI: https://doi.org/10.25035/pad.2020.02.004

Available at: https://scholarworks.bgsu.edu/pad/vol6/iss2/4

This Main Article is brought to you for free and open access by the Journals at ScholarWorks@BGSU. It has been accepted for inclusion in Personnel Assessment and Decisions by an authorized editor of ScholarWorks@BGSU. 


\title{
Does Feedback InCReAse Decision Aid Use Among Hiring Professionals?
}

\author{
Aneeqa Thiele ${ }^{1}$, Alexander T. Jackson ${ }^{1}$, Stacey M. Stremic ${ }^{1}$, and \\ Satoris S. Howes ${ }^{2}$
}

1. Middle Tennessee State University

2. Oregon State University

\section{KEYWORDS}

decision aids, feedback, personnel selection
ABSTRACT

We examined the influence of formative and outcome feedback on people's reliance on decision aids. Decision aids are tools that managers can use to increase the accuracy of their hiring decisions. In our study, participants were asked to make 20 different hiring decisions and make predictions of a candidate's performance on the job, with the option of using a decision aid formula. We manipulated whether participants received feedback on the accuracy of their predictions, the accuracy of the decision aid's predictions, or both. The results demonstrated that feedback failed to have a significant impact on decision aid use for both hiring choice and performance predictions. Our findings suggest that the relationship between feedback and decision aid is weak, and that feedback does not meaningfully affect the use of decision aids.
Human resource practices are of key importance to organizations, with the selection, recruitment, and training of new employees costing companies billions of dollars every year. Researchers have estimated that quality selection practices can save companies millions of dollars every year (Chang et al., 2013). Along these lines, decision aids, such as statistical aids, can help hiring managers make better hiring decisions in the workplace (Highhouse, 2008). However, a large body of literature has shown that managers resist the use of decision aids and prefer to rely on intuitive methods, despite these methods yielding poor predictive validity (Kuncel et al., 2013; Fernandez \& Pougnet, 2018). In fact, taking an algorithmic approach to decision making outperforms intuitive decision making by more than $50 \%$ in predicting job performance (Kuncel et al., 2013). Nevertheless, practitioners continue to prefer the use of intuition over the use of decision aids.

In the present paper, we sought to determine whether providing feedback on the (in)accuracy of one's performance predictions and hiring choices as well as the accuracy of a decision aid can help persuade hiring managers to use decision aids. Feedback is a powerful tool used by managers to improve performance, strengthen communication between employees and managers, address work behaviors, and set goals (i.e., performance reviews, 360-degree appraisal, etc.; Shute, 2008). Furthermore, feedback can effectively be used to remove ambiguity and provide solutions to help people make better decisions in the future (Procelli \& Delgado, 2017; Shute, 2008). We predict that if hiring managers receive such feedback when they use decision aids, they can learn how to use decision aids appropriately. Further, we expected that information regarding the (in)accuracy of participants' hiring predictions would produce sufficient motivation for participants to improve their performance. By providing feedback that shows that the decision aid outperforms human judgment, we hoped to make people more likely to use decision aids, thereby enhancing the quality of their decisions.

\section{Using Decision Aids for Selection}

Hiring managers make selection decisions by collecting a considerable amount of information about applicants in the form of job applications, biodata, tests, interviews, résumés, job simulations, and job references. It can be difficult to use all of this information appropriately and select the best candidate (Kuncel et al., 2013). This challenge can be overcome, however, through the use of decision aids.

\footnotetext{
Corresponding author:

Alexander T. Jackson

Middle Tennessee State University

Box 87, 1301 E. Main St., Murfreesboro, TN 37132

Email: Alexander.jackson@mtsu.edu
} 
Decision aids can help decision makers analyze information in depth by helping them make judgments that are based on a predetermined formula (Slaughter \& Kausel, 2014). This formula helps reduce error in the prediction of employee performance. Examples include simple unit weighting or predictors, mechanical combinations of predictors using linear regression, and decision trees (Highhouse, 2002). Decision aids use a mechanical or algorithmic approach that applies the same weight to each applicant's score rather than using human judgment or intuition to select an applicant. This approach significantly increases both consistency and accuracy within decisions because it establishes a standard for the hiring process, which can help managers collect and use applicant information appropriately (Slaughter \& Kausel, 2014).

However, people are hesitant to rely on decision aids (Rynes et al., 2018). For example, Dongen and Maanen (2013) found that people easily justify their errors in judgment, yet they heavily weigh any errors from decision aids. In their study, people consistently underestimated the reliability of the decision aid in comparison to their own reliability in decision making. Although participants attributed their errors to uncontrollable and justifiable causes, they believed that decision aid errors were more likely explained by stable, dispositional factors. Thus, it appears people tend to trust and believe their judgments are superior to other methods (Slaughter \& Kausel, 2014).

A recent recommendation to encourage the use of decision aids is to offer decision support based on statistical predictions (Highhouse et al., 2017). However, because people are hesitant to rely solely on mechanical methods (Slaughter \& Kausel, 2014), support may need to come in the form of unambiguous feedback about their hiring choices (Shute, 2008). Thus, we sought to encourage the use of decision aids first by providing the decision aid and asking decision makers to make specific predictions about a candidate's performance. We then provided a combination of formative feedback (i.e., feedback given in a specific, timely, supportive, and nonevaluative way to signal a gap between a person's current performance and a desired level of performance) and outcome feedback (i.e., informative feedback that simply relays information about performance outcomes) about the participant's predictions and the decision aid's predictions. We also provide support by giving participants feedback about their performance predictions.

\section{Using Formative Feedback and Outcome Feedback to Influence Managers}

Feedback is used in management practices to help people accomplish their tasks and provide information about how they are doing, how accurately they are doing their jobs, and the adequacy of their work behaviors (DeNisi \&
Kluger, 2000). Feedback provides people information about their performance and is a significant factor in motivating learning (Shute, 2008). Feedback can often take two forms: formative feedback and outcome feedback. Outcome feedback requires people to receive feedback about the accuracy of their decisions or selections (Geister et al., 2006), such as feedback that informs employees about their work performance (i.e., performance appraisals; Tuttle \& Stocks, 1997). Outcome feedback is an effective form of feedback for changing employee behavior (Taylor et al., 1996). Outcome feedback motivates people to improve their performance by first shedding light on their performance, which in turn influences their efforts and leads to an increase in performance (Geister et al., 2006). Indeed, receiving information about performance, such as the outcome and ways to improve performance, has been shown to be a motivational factor to foster perseverance (Roney et al., 1995). Porcelli and Delgado (2017) noted that people's decision-making performance improved after repeated exposure to positive and negative feedback, especially in conditions in which participants received positive outcome feedback.

Although outcome feedback is a useful tool that provides information about employee performance, it does not provide solutions to make appropriate adjustments to better their performance. In fact, people who only receive outcome feedback when completing complex and unstructured tasks may make inappropriate adjustments (Earley et al., 1990). It seems the missing element that would aid in improvement lies with formative feedback, in which information is communicated with the intent to alter a person's thinking or behavior to improve learning (Shute, 2008). Indeed, formative feedback is given in a specific and nonevaluative way, and is used by managers as a way to reduce the uncertainty inherent in making hiring decisions by providing a set of specific areas (e.g., reducing personal bias, being more consistent with ratings) that decision makers can focus on to improve their decision making. This is important for selection decisions because people tend to over-rely on their own intuitive judgments when making hiring decisions, especially when the consequences of hiring decisions are not immediate and decision makers do not receive feedback (Brown, 2006).

Providing specific informative feedback, such as that inherent with formative feedback, along with outcome feedback has been shown to substantially increase quality decision making and information search (Earley et al., 1990; Geister, et al., 2006). Moreover, in support of formative feedback, which aids in reducing uncertainty, Porcelli and Delgado (2017) found decisions made under uncertainty led to more risk taking and financially disadvantageous choices. Research suggests that any instructional activity that requires people to search for an explanation to a problem impairs learning because it increases one's cognitive load. 
This issue is especially salient when people have limited prior knowledge about that particular problem and they have no guidance (Moreno, 2004).

Kuncel (2008) argued that selection experts are rarely given information about the accuracy of their decisions and giving "hard-to-ignore" feedback regarding decision-making accuracy could help reduce overconfidence and lead to better decision making (p. 345). Outcome feedback can be a purposeful way to inform people about the accuracy of their predictions, whereas formative feedback can help people learn how to use decision aids more effectively. Thus, providing informative feedback on how one is performing (outcome feedback) in a way that is specific, timely, nonevaluative, and indicative of what changes may be needed (formative feedback) can influence people's reliance on decision aids because it provides unambiguous information regarding their performance (i.e., accuracy) and how to improve (i.e., utilize decision aid). Upon receiving performance feedback, decision makers should be motivated to make better decisions and reduce their prediction inaccuracies (Shute, 2008).

Within this study, we provide participants with feedback about their own accuracy and feedback about the accuracy of the decision aid. Separately, each of these pieces of information represent outcome feedback in that they inform the individual about his or her own performance and the performance of the decision aid. As such, we expect individuals who received either type of feedback individually to rely on decision aids more, because they will see that their performance is low (and therefore necessitates the decision aid) or the decision aid accuracy is high (and justifies its use). In combination, however, we argue that the impact on decision aid reliance is even greater, as the feedback not only reflects outcome feedback but is also formative because it should be much clearer (i.e., less ambiguous) regarding what should be done. The combination of the feedback, which is specific, timely, and nonevaluative, will demonstrate that their accuracy is low and the decision aid is high, thereby eliminating any uncertainty that could still exist. With only outcome feedback on their own accuracy (or only on the decision aid accuracy), it is unclear whether the decision aid (or their own accuracy) may have been better or worse. The combination of the two pieces of information, however, leaves little question as to which is optimal, and therefore individuals who receive feedback on both their own accuracy and the decision aid will be more likely to rely on the decision aid. As such, we hypothesize the following:

Hypothesis 1: Individuals who receive outcome feedback about their accuracy will rely on the decision aid more than will individuals who receive no such feedback.
Hypothesis 2: Individuals who receive outcome feedback about the accuracy of the decision aid will rely on the decision aid more than individuals who receive no such feedback.

Hypothesis 3: There will be an interaction between outcome feedback about one's accuracy and outcome feedback about the accuracy of the decision aid, such that those who receive both forms of feedback will be most likely to rely on the decision aid.

\section{METHOD}

\section{Participants}

Hiring professionals were recruited from Amazon's Mechanical Turk (MTurk) and Qualtrics research panels. To participate in the study, individuals had to meet the following screening procedures: English was their native language, they must have had at least 2 years of hiring experience, and they must currently have a job. Next, to ensure that our data were of sound quality, our survey contained a total of five attention check questions dispersed throughout the survey. If a participant failed any one of the five, their data were excluded. Our final sample consisted of 900 working professionals $(60.1 \%$ female, $74.9 \%$ White, non-Hispanic). Approximately $70.4 \%$ of participants had a 2 -year college degree or higher. The average age of participants was 36.85 years $(S D=10.88)$. The median years of hiring experience was 5 years.

\section{Materials and Procedure}

Participants were presented with selection information for 20 pairs of applicants using a modified version of the decision aid task used by Jackson et al. (2019). The selection criteria given to the participants consisted of the candidates' scores (Candidate A and Candidate B) on conscientiousness, cognitive ability, and an unstructured interview. Participants were also provided background information regarding the predictors. For example, participants were told that cognitive ability is considered a good predictor, conscientiousness is a moderate predictor, and the unstructured interview is a weak predictor of performance.

The participants, who were asked to play the role of a hiring manager, were given a decision aid in the form of a formula. An example was provided that fully demonstrated how the decision aid should be used. For each decision in the hiring task, participants were reminded what the decision aid formula was and were provided with the weighted scores for each candidate. This essentially reduced some of the mental burdens of multiplying the candidates' scores by the weights in the decision aid. If participants elected to use the decision aid, they merely needed to sum the weighted scores for each candidate to get the candidate's predicted 
performance. Last, the participants were asked to answer, based on the prehiring information, to estimate how well each candidate would perform on a scale of $0 \%$ ("will perform worse than all other employees") to $100 \%$ ("will perform better than all other employees") and select which candidate should be hired. Eventual performance of the job candidates was pre-determined using the same approach as Jackson et al. (2019). ${ }^{1}$ An example of the stimuli presented to participants is displayed in Appendix A.

As previously mentioned, previous researchers have suggested that one reason people do not use decision aids is that people do not trust the decision aids (Kuncel, 2008). Therefore, we sought to control for initial levels of trust in the decision aid in our analyses. Before completing the decision task, participants were asked to indicate their level of trust in the decision aid. Trust was measured with five items using a 7-point Likert scale ( 1 = "strongly disagree", 7 = "strongly agree"). Sample items stated, "The prediction formula is dependable" and "I can trust the prediction formula." The internal consistency reliability for the scale was $\alpha=.87$. Participants were then asked to make the 20 hiring decisions. Using the information provided by the decision aid or not, participants then made a hiring choice between the candidates and made a prediction about each candidate's future performance on the job.

Feedback was manipulated by randomly assigning participants to receive outcome feedback (or not receive outcome feedback) regarding the accuracy of the decision. Participants were randomly assigned to receive individual outcome feedback (i.e., feedback about the accuracy of their performance predictions) or not receive individual outcome feedback. Additionally, participants were randomly assigned to receive decision aid outcome feedback (i.e., feedback about the accuracy of the decision aid's predictions) or not receive decision aid outcome feedback. The feedback itself was presented in terms of how many percentage points off the decision aid or the participant were in estimating the candidates' eventual performance. An example of what the participants saw is provided in Appendix A. Thus, in our study, there were two separate feedback manipulations (one for feedback about the participant's performance predictions and one for feedback about the decision aid's predictions), and our study used a 2 (individual outcome feedback provided or not) x 2 (decision aid outcome feedback provided or not) repeated measures design, with participants making a total of 20 decisions.

\section{RESULTS}

To evaluate the internal validity of our study, we examined the relationship between participants' performance predictions and their hiring choices. This enabled us to determine whether participants understood the task by showing whether participants selected the candidate that they predicted would perform better. Candidate performance predictions was calculated as the difference in the participants' performance predictions between candidate B and candidate A (candidate B - candidate A). If the score was greater than zero, the participant predicted that candidate $\mathrm{B}$ would perform better. If the score was less than zero, the participant predicted that candidate A would perform better. Participants' hiring choice was coded as candidate $\mathrm{A}=-1$ and candidate $\mathrm{B}=1$. As can be seen in Table 1 in Appendix $\mathrm{B}$, the correlation between participant hiring choice and participant performance predictions is quite large $(r=.663)$, showing that participants did tend to select the candidate they rated more favorably.

To test our hypotheses, decision aid use was operationalized in two ways. First, we operationalized decision aid use in terms of whether participants' hiring choices matched the choices of the decision aid. Second, we operationalized decision aid use as the extent to which participants' performance predictions matched the performance predictions of the decision aid. Using multiple operationalizations of the decision aid use allowed us to examine whether differences would emerge in the two behavioral responses of participants.

\section{Match in Hiring Choices}

To determine whether feedback had a significant impact match in hiring choice, we utilized the generalized linear mixed-effects modeling package (glmer) in R (Bates et al., 2014). Specifically, we conducted a repeated-measures logistic regression with match in hiring choice (a binomial outcome) as the dependent variable. First, we controlled for the data source (MTurk vs. Qualtrics), prior experience with hiring decisions, education level, and trust in the decision aid by entering these variables as fixed effects. Then we entered individual outcome feedback, decision aid outcome feedback, and trial (our time variable), and their interactions as fixed effects. To reduce any multicollinearity individual outcome feedback and decision aid outcome feedback were centered using effects coding, and trial was centered using mean centering. Table 1 in Appendix B displays the correlation matrix for all of the study variables.

Overall, we found that there was not a significant main effect of individual outcome feedback $(B=0.01, z=0.68, p$ $=.49)$, decision aid outcome feedback $(B=-0.01, z=-0.37$, $p=.71)$, or trial $(B=0.002, z=0.62, p=.54)$. Furthermore,

1 Eventual performance was determined using the formula $y$ $=$ round $\left(\right.$ logistic $\left(\right.$ logistic percent $\left(.50 * x_{1}+.40 * x_{2}+.10 * x_{3}\right)+$ $\left.x_{r} \sim N(0,1)\right) * 100$, whereby $y=$ eventual performance, $x_{1}=$ cognitive ability score of the candidate, $x_{2}=$ conscientiousness score of the candidate, $x_{3}=$ interview score of the candidate, and $x_{r} \sim N(0,1)=$ error, created by selecting a random value from a normal distribution with an average of 0 and a standard deviation of 1 (see Jackson et al., 2019). 
none of the two-way interactions or the three-way interaction were significant. This suggests that neither outcome feedback about the accuracy of one's own decisions nor outcome feedback about the accuracy of a decision aid impacts whether one is likely to make hiring decisions that match a decision aid and thus utilize the decision aid. Additionally, it appears that these effects do not significantly change over time.

\section{Match in Performance Predictions}

To calculate the match in performance predictions, we calculated the absolute difference between participants' predictions and the decision aid's predictions for both candidates. This yielded a measure of the magnitude of the difference in performance predictions for each candidate. However, because scores closer to zero would indicate a greater degree of match, the difference scores were reversed by subtracting the difference scores from 100 . Then, because difference scores are not normally distributed, we performed a $\log$ transformation on the absolute differences. Thus, scores closer to zero indicated a greater degree of mismatch in predictions, whereas scores greater than zero indicated increasing degrees of match in predictions. For ease of explanation, we will simply refer to this as the match in performance predictions. To test whether individual outcome feedback or decision aid outcome feedback affected the match in performance predictions between participants and the decision aid, we conducted a repeated-measures linear regression using the linear mixed-effects modeling package (lmer) in R (Bates et al., 2014). We entered the match in performance predictions as the dependent variable. We then controlled for prior experience with hiring decisions, education level, and trust in the decision aid by entering these variables as fixed effects. Then we entered individual outcome feedback, decision aid outcome feedback, and trial (our time variable), and their interactions as fixed effects. These variables were centered using the same procedures as described above.

We found no significant main effects for individual outcome feedback $(B=-0.003, t(5,110)=-0.81, p=.42)$ or decision aid outcome feedback $(B=0.002, t(5,092)=0.59, p$ $=.55)$. However, there was a significant main effect of trial $(B=-.001, t(35,100)=-2.68, p<.01)$. This suggests that as time progressed, participants' performance predictions were less likely to match the decision aid's predictions. Interestingly, there was a significant two-way interaction between individual outcome feedback and trial $(B=0.001, t(35,100)$ $=2.37, p=.02$ ). Figure 1 (displayed in Appendix B) shows the two-way interaction between individual feedback and time. As can be seen in Figure 1, for those who received individual outcome feedback, the match in their performance predictions with the decision aid's predictions did not change significantly over time. However, for those who did not receive individual outcome feedback, the match in performance predictions decreased over the 20 decisions. This suggests that when people do not receive the feedback about their predictions they may be less likely to rely on decision aids over time.

\section{DISCUSSION}

Managers want the autonomy to trust their intuition for hiring decisions. Yet, research shows that intuition is a poor method for making decisions. Intuitive decisions are often biased, lead to poor selection decisions (e.g., inaccurate predictions, inconsistent standards, etc.), poor performance among employees, and significant costs for the organization. The divide between what is often practiced (subjective intuitive methods) and what is recommended (statistical-based methods) still continues to exist.

This study examined whether feedback would influence people's utilization of decision aids. Our results showed that people who received outcome feedback about the accuracy of their decisions did not rely on the decision aid more than people who received no feedback, failing to support Hypothesis 1. We also did not find support for Hypothesis 2 or 3. Specifically, our results indicated that people who received outcome feedback about the accuracy of the decision aid did not rely on the decision aid more than people who received no feedback, and there was no interaction between outcome feedback about a person's accuracy and outcome feedback on the accuracy of the decision aid. Furthermore, we only found two significant effects, neither of which were hypothesized. When decision aid use was operationalized as a greater degree of match in performance predictions, we found a main effect of trial, which suggests that participants were less likely to make performance predictions that matched the decision aid's over time. In other words, participants seemingly relied less on the decision aid to make their performance predictions overtime. Further, we found that trial interacted with individual outcome feedback, such that the decline in the match in performance predictions (and reliance on the decision aid) only appeared to occur for the participants who did not receive any feedback about the accuracy of their performance predictions. For those who did receive feedback, their match in performance predictions did not change throughout the 20 trials, indicating that they did not increase nor decrease their use of the decision aid. It is worth noting that the sizes of our effects are essentially near zero, so despite being significant, these results may not hold practical significance.

Our results were surprising given the profound effects that have been found regarding the power of feedback (Shute, 2008; Slaughter \& Kausel, 2014). Indeed, Dietvorst et al. (2014) found that when people saw a decision aid make a mistake, they were less likely to rely on the decision aid in the future, even if the error was smaller than human errors. Our findings seem to contradict previous research, 
though it is unclear why this is. One possible reason is our sample comprised actual hiring managers accustomed to engaging in tasks similar to the one they experienced in this study. That is, previous research has focused on student samples or online samples of individuals without regard to their working status (cf. Dietvorst et al., 2014). It could be the case that the previous findings do not extend to other populations. Our screening procedures to ensure that we were recruiting only individuals with at least 2 years of hiring experience resulted in obtaining participants with a wide range of applied human resource backgrounds and clear experience with the task at hand. As such, one might expect that experienced individuals doing tasks relevant to their expertise rely more heavily on their own intuition and distrust decision aids more than originally expected. Although this was not the purpose of our study, our correlation table shows no significant relationship between trust in the decision aid and hiring experience.

An interesting finding is the significant relationship between match in hiring choice and match in performance predictions. This relationship is substantially smaller (albeit still positive and significant) than the relationship between the participant's performance predictions (Candidate B vs. Candidate A) and their hiring choice. Although we expected the relationship between the participant's performance predictions and their hiring choice to be large, indicating internal validity, we were somewhat surprised by the difference in the magnitude of the relationships. One possible explanation of this difference is that while people may have made similar choices as the decision aid, their performance predictions may have been quite different than the predictions made by the decision aid. This would be the case if people were simply estimating performance or relying on some form of heuristic when making predictions. The absolute value of their predictions compared to the predictions of the decision aid may be different, but the relative difference between Candidate A and Candidate B may have been the same. This would result in participants making somewhat different performance predictions than the decision aid but making similar hiring choices as the decision aid.

\section{Limitations}

One possible limitation of our studies was the use of MTurk and Qualtrics to recruit participants. We utilized crucial inclusion criteria (e.g., minimum of 2 years of hiring experience) and multiple attention checks throughout the study. However, there is some controversy over the quality of online sampling techniques (Smith et al., 2016). Future studies could use other sampling techniques to replicate previous findings on the influence of feedback on decision aid utilization. However, there is a substantial amount of research that suggests that MTurk samples (and those similar, such as Qualtrics panels) are equivalent to other types of sampling. Hauser and Schwarz (2016) found that
MTurk participants were more attentive than subject pool participants and, in some cases, identical to other methods of sampling (Casler et al., 2013). Also, MTurk samples can provide a greater diversity of demographics (Dworkin et al., 2016).

In our study, we measured hiring experience, not previous experience with decision aids. We suggest that experience with and/or previous exposure to decision aids might influence participants' level of trust and use of the decision aid. Dietvorst et al. (2016) suggested that experience with decision aids can impact an individual's' use of them. If participants had negative experiences of perceptions of the decision aid, it may have lessened the influence of feedback on the use of the decision aid. More research is needed to explore these possibilities.

A final limitation of this study was that we informed participants at the outset of the study what the best and worst predictors of job performance are. This may have inadvertently cued participants to attend to the predictors in the manner we specified. Further, this may, at least partially, explain why we found such little variation between the conditions and across time. However, the small positive correlation between the match in performance predictions and match in hiring choices suggests that participants were not fully utilizing the cue information we provided. If they had been, we might have observed a larger correlation. Further, given that the hypotheses focused explicitly on whether feedback would impact the use of the decision aid, we do not think that informing the participants about the relative importance of the predictors would impact the results of the hypotheses.

\section{Practical Implications}

A key practical implication from our study is that if organizations want increase hiring managers' reliance on decision aids, they should not rely exclusively on feedback. We found that providing simple, clear feedback about the accuracy of performance predictions did not increase reliance on decision aids. Although this is not what we expected nor what we hoped, these findings do help researchers and managers narrow down the search for ways to shift decision makers away from their own heuristics, biases, and intuition toward using statistically supported and superior decision aids. One possible avenue for future research is to examine other forms of feedback. Perhaps providing more instructional information on how to improve, beyond prediction inaccuracies, may lead to greater reliance on the decision aids.

\section{Conclusion}

The selection process within the organization remains a critical aspect of the organization's health and growth. It is undoubtedly clear that managers overly rely on intuitive 
methods for hiring decisions. Consequently, it is crucial to explore methods to decrease their reliance on personal judgments and increase their likelihood to use more reliable methods of hiring, such as decision aids. Perhaps the biggest obstacle to decision aid acceptance is the presence of algorithm aversion (Dietvorst et al., 2014). Unfortunately, hiring managers ignore superior forecasting methods and prefer intuitive methods for making decisions in the workplace. Decision aids, such as the statistical formula used in this study, lack the autonomy and familiarity of other common methods (e.g., intuition). The loss of predictive validity when using intuition as the basis for selection decisions is too great a cost for organizations. Further, organizations should not only want consistent methods of selection to protect themselves legally, but they should strive for quality methods of hiring for better accuracy and results (i.e., better employee performance). Thus, we must continue to search for ways to guide hiring professional away from subjective methods and toward effective and scientifically based methods. If feedback does not increase the use of decision aids, then we must find other, more effective ways to persuade hiring professionals.

\section{REFERENCES}

Bates, D., Machler, M., Bolker, B. M., \& Walker, S. C. (2014). Ime4: Linear mixed-effects models using Eigen and S4 [Computer software]. Retrieved from http://cran.r-project.org/package $=$ Ime 4

Brown, J. C. (2006). The effects of behavioral and outcomes feedback on prudent decision-making under conditions of present and future uncertainty. Judgment and Decision Making, 1(1), 76-85.

Casler, K., Bickel, L., \& Hackett, E. (2013). Separate but equal? A comparison of participants and data gathered via Amazon's MTurk, social media, and face-to-face behavioral testing. Computers in Human Behavior, 29, 2156-2160. Doi: 10.1016/j.chb.2013.05.009

Chang, W. A., Wang, Y., \& Huang, T. (2013). Work design-related antecedents of turnover intention: A multilevel approach. Human Resource Management, 52(1), 1-26. Doi: 10.1002/ hrm. 21515

DeNisi, A. S., \& Kluger, A. N. (2000). Feedback effectiveness: Can 360-degree appraisals be improved? Academy of Management Executive, 14(1), 129-139. Doi: 10.5465/ ame.2000.2909845.

Dietvorst, B. J. Simmons, J. P., \& Massey, C. (2014). Algorithm aversion: People erroneously avoid algorithms after seeing them err. Journal of Experimental Psychology: General, 144(1), 114-126. Doi: 10.1037/xge0000033

Dietvorst, B. J., Simmons, J. P., \& Massey, C. (2016). Overcoming algorithm aversion: People will use imperfect algorithms if they can (even slightly) modify them. Management Science, 64(3), 1155-1170. Doi: 10.1287/mnsc.2016.2643

Dongen, K. V., \& Maanen, P. V. (2013). A framework for explaining reliance on decision aids. Journal of Human-Computer
Studies, 71, 410-424. Doi: 10.1016/j.ijhcs.2012.10.018.

Dworkin, J., Hessel, H., Gliske, K., \& Rudi, J. H. (2016). A comparison of three online recruitment strategies for engaging parents. Interdisciplinary Journal of Applied Family Studies, 65, 550-561. Doi: 10.1111/fare.12206.

Earley, P. C., Northcraft, G. B., Lee, C., \& Lituchy, T. R (1990). Impact of process and outcome feedback on the relation of goal setting to task performance. Academy of Management Journal, 33(1), 87-105. Doi: 10.5465/256353

Fernandez, S., \& Pougnet, S. (2018). Is there structure in the selection interview? Evidence from hotels in Switzerland. Journal of Human Resources in Hospitality \& Tourism, 17(1), 80-97. Doi: 10.1080/15332845.2017.1328262

Geister, S., Konradt, U., \& Hertel, G. (2006). Effects of process feedback on motivation, satisfaction, and performance in virtual teams. Small Group Research, 37(5), 459-489. Doi: $10.1177 / 1046496406292337$

Hauser, D. J., \& Schwarz, N. (2016). Attentive Turkers: MTurk participants perform better on online attention checks than do subject pool participants. Behavioral Research, 48, 400-407. Doi: 10.3758/s13428-015-0578-z.

Highhouse, S. (2002). Assessing the candidate as a whole: A historical and critical analysis of individual psychological assessment for personnel decision making. Personnel Selection, 55(2), 363-396. Doi: 10.1111/j.1744-6570.2002. tb00114.x

Highhouse, S. (2008). Stubborn reliance on intuition and subjectivity in employee selection. Industrial and Organizational Psychology, 1(3), 333-342. Doi: 10.1111/j.17549434.2008.00058.x

Highhouse, S., Brooks, M. E., Nesnidol, S., \& Sim, S. (2017). Is a .51 validity coefficient good? Value sensitivity for interview validity. International Journal of Selection and Assessment, 25(4), 383-389. Doi: 10.1111/ijsa.12192

Jackson, A. T., Young, M. E., Howes, S. S., Knight, P. A., \& Reichin, S. L. (2019). Examining factors influencing use of a decision aid in personnel selection. Personnel Assessment and Decisions, 5(1). Doi: 10.25035/pad.2019.01.001

Kuncel, N. R. (2008). Some new (and old) suggestions for improving personnel selection. Industrial and Organizational Psychology: Perspectives on Science and Practice, 1(3), 343-346. Doi: 10.1111/j.1754-9434.2008.00059.x.

Kuncel, N. R., Klieger, D. M., Connelly, B. S. \& Ones, D. S. (2013). Mechanical versus clinical data combination in selection and admissions decisions: A meta-analysis. Journal of Applied Psychology, 98, 1060-1072. Doi:10.1037/a0034156

Moreno, R. (2004). Decreasing cognitive load for novice students: Effects of explanatory versus corrective feedback in discovery-based multimedia. Instructional Science, 32(1-2), 99-113. Doi: 10.1023/B:TRUC.0000021811.66966.1d

Porcelli, A. J., \& Delgado, M. R. (2017). Stress and decision making: Effects on valuation, learning, and risk-taking. Current Opinion in Behavioral Sciences, 14, 33-39. Doi: 10.1016/ j.cobeha.2016.11.015

Roney, C. J. R., Higgins, E. T., \& Shah, J. (1995). Goals and framing: How outcome focus influences motivation and emotion. Personality and Social Psychology Bulletin, 21(11), 11511160. Doi: 10.1177/01461672952111003.

Rynes, S. L., Colbert, A. E., \& O'Boyle, E. H. (2018). When the "best 
available evidence" doesn't win: How doubts about science and scientists threaten the future of evidence-based management. Journal of Management, 44(8), 2995-3010. Doi: $10.1177 / 0149206318796934$

Shute, V. J. (2008). Focus on formative feedback. Review of Educational Research, 78(1), 153-189. Doi: 10.3102/0034654307313795

Smith, S. M., Roster, C. A., Golden, L. L., \& Albaum, G. S. (2016). A multi-group analysis of online survey respondent data quality: Comparing a regular USA consumer panel to MTurk samples. Journal of Business Research, 69, 3139-3148. Doi: 10.1016/j.jbusres.2015.12.002

Slaughter, J. E., \& Kausel, E. E. (2014). Employee selection decisions. In S. Highhouse, R. S. Dalal, \& E. Salas (Eds.), Judgment and decision making at work (pp. 57-79). New York, NY: Routledge.

Taylor III, L. A., Hall, P. H., Cosier, R. A., \& Goodwin, V. L. (1996). Outcome feedback effects on risk propensity in an MCPLP task. Journal of Management, 22(2), 299-311. Doi: 10.1016/ S0149-2063(96)90050-1

Tuttle, B., \& Stocks, M. H. (1997). The effects of task information and outcome feedback on individuals' insight into their decision models. Decision Sciences, 28, 421-442. Doi: 10.1111/ j.1540-5915.1997.tb01317.x

RECEIVED 02/01/19 ACCEPTED 04/04/20 


\section{Appendix A}

Example Materials

\section{Example of Decision Stimuli}

Below is the information for two candidates. Use this information to predict each applicant's job performance and identify which candidate the organization should hire.

\begin{tabular}{|c|c|c|c|}
\cline { 2 - 4 } \multicolumn{1}{c|}{} & $\begin{array}{c}\text { Cognitive Ability Test } \\
\text { percentile rank }\end{array}$ & $\begin{array}{c}\text { Conscientiousness Test } \\
\text { percentile rank }\end{array}$ & $\begin{array}{c}\text { Unstructured Interview } \\
\text { percentile rank }\end{array}$ \\
\hline Candidate A & 73 & 55 & 19 \\
\hline Candidate B & 86 & 59 & 98 \\
\hline
\end{tabular}

(Note: Percentile is the percentage of individuals who score less than the candidate. For example, a percentile score of 50 on the cognitive ability test means that the candidate performed better than $50 \%$ of the other individuals).

Recall that the prediction formula was:

$0.50 \times($ cognitive ability score $)+0.40 \times($ conscientiousness score $)+0.10 \times($ unstructured interview score $)=$ predicted job performance

Based on the scores for each candidate, the formula for each candidate is:

Candidate A:

$36.5+22+1.9=$ predicted job performance

Candidate B:

$43+23.6+9.8=$ predicted job performance

\section{Example of Hiring Choice and Performance Predictions}

Based on this prehiring information, on a scale of $0 \%$ (will perform worse than all other employees) to $100 \%$ (will perform better than all other employees), how well do you think each candidate will perform on the job?

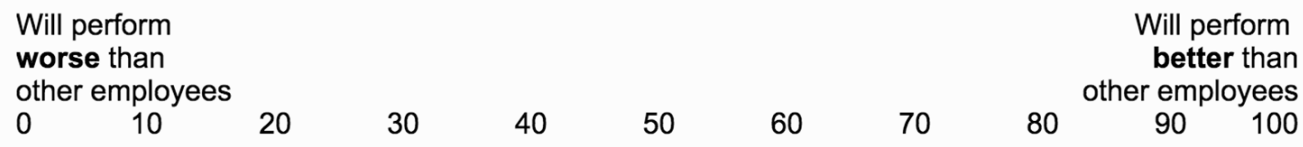

Predicted job performance of Candidate A:

Predicted job performance of Candidate B:

Based on this pre-hiring information, which candidate should the organization hire?

Candidate A

Candidate B 


\section{Example of the feedback provided to participants}

In the previous decision, you estimated that the candidates' performance would be:

Candidate A: $\mathbf{5 0 \%}$

Candidate B: 50\%

In the previous decision, the formula estimated that the candidates' performance would be:

Candidate A: $49.1 \%$

Candidate B: $25.1 \%$

Once both candidates were hired, their actual job performance was:

Candidate A: $\mathbf{5 6 \%}$

Candidate B: 3\%

Your prediction for Candidate A was off by 6 percentage points.

Your prediction for Candidate B was off by 47 percentage points.

The prediction by the formula for Candidate A was off by 6.9 percentage points

The prediction by the formula for Candidate B was off by 22.1 percentage points 
Appendix B

Tables \& Figures

TABLE 1.

Intercorrelations Between the Study Variables

\begin{tabular}{|c|c|c|c|c|c|c|c|c|c|c|}
\hline & 1 & 2 & 3 & 4 & 5 & 6 & 7 & 8 & 9 & 10 \\
\hline 1. Data source & -- & & & & & & & & & \\
\hline 2. Education & .033 & -- & & & & & & & & \\
\hline 3. Hiring experience & -.166 & .038 & -- & & & & & & & \\
\hline 4. Trust & .009 & .015 & -.005 & -- & & & & & & \\
\hline 5. Individual feedback & .044 & -.018 & -.034 & -.026 & -- & & & & & \\
\hline 6. Decision aid feedback & .052 & .016 & -.065 & -.024 & .009 & -- & & & & \\
\hline 7. Trial & $<-.001$ & $<-.001$ & $<-.001$ & $<-.001$ & $<.001$ & $<.001$ & -- & & & \\
\hline 8. Match in hiring choices & -.001 & -.003 & -.004 & .001 & .005 & -.003 & .005 & -- & & \\
\hline 9. Match in performance predictions & .028 & -.001 & -.024 & -.028 & .015 & .016 & -.014 & .138 & -- & \\
\hline 10. Participant hiring choice & .009 & .004 & $<-.001$ & .002 & .005 & .004 & .182 & $<.001$ & -.019 & -- \\
\hline 11. Participant performance predictions & .001 & .002 & -.005 & .014 & .014 & .012 & .145 & $<.001$ & .005 & .663 \\
\hline
\end{tabular}

Note. Data source was coded as $-1=$ Qualtrics and $1=$ MTurk. Education was coded as $1=$ less than high school, $2=$ high school/GED, 3 = some college, $4=2$-year college degree, $5=4$-year college degree, $6=$ master's degree, $7=$ doctoral degree, $8=$ postgraduate professional degree. Hire choice was coded as $-1=$ Candidate A and $1=$ Candidate B. Bolded values are significant at $p<.05$.

\section{FIGURE 1.}

Two-way interaction among individual outcome feedback, decision aid outcome feedback, and trial in Study 1. Note that the $y$-axis has been rescaled to allow for ease of display and interpretation. Error bars represent $+/-1$ standard error.

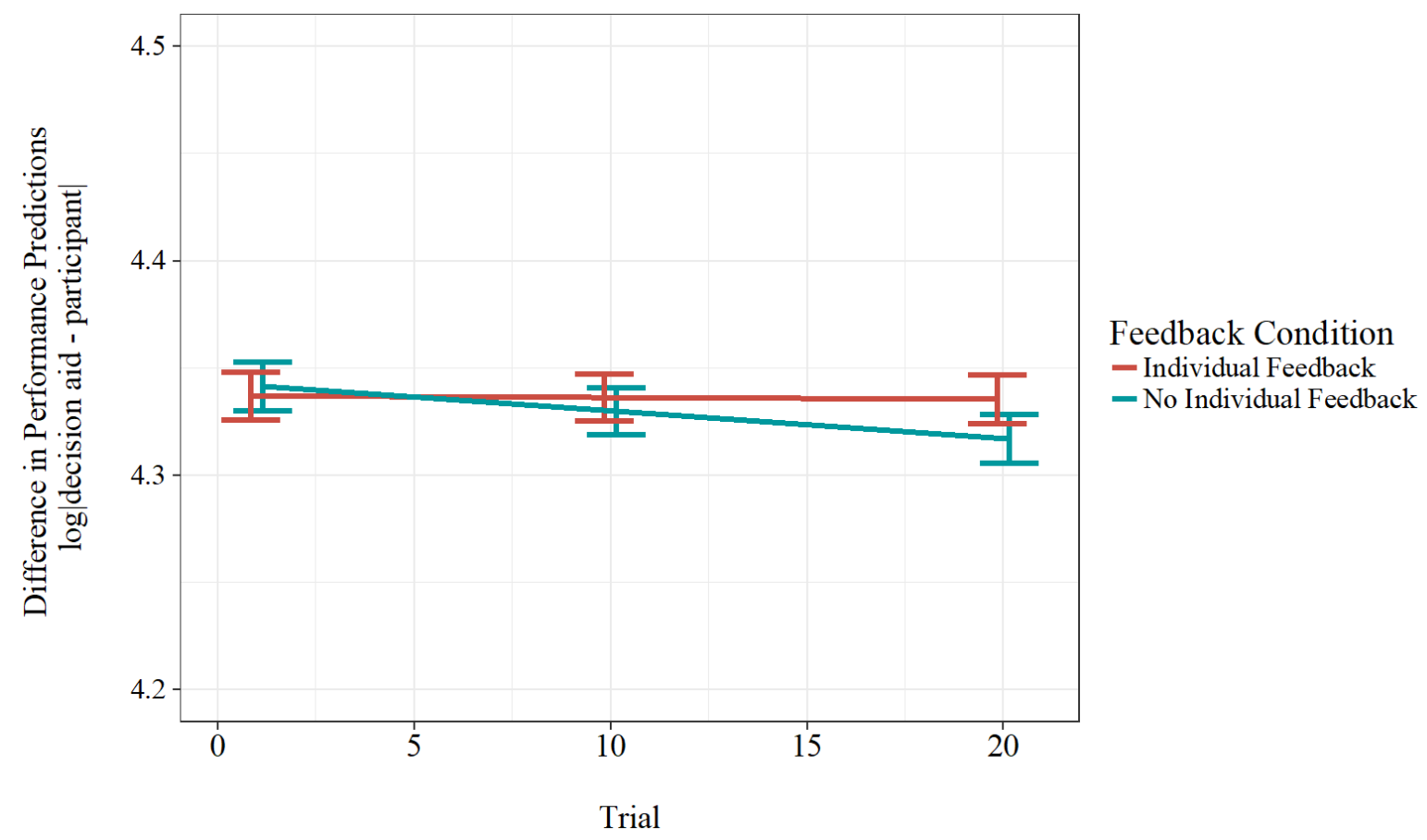

